\title{
On the criterion for Bose-Einstein condensation for particles in traps
}

\author{
C. J. Pethick ${ }^{1}$ and L. P. Pitaevskii ${ }^{2,3}$ \\ ${ }^{1}$ NORDITA, Blegdamsvej 17, DK-2100 Copenhagen Ø, Denmark \\ ${ }^{2}$ Department of Physics, University of Trento, I-38050 Povo (Trento), Italy \\ ${ }^{3}$ Kapitza Institute for Physical Problems, 117334, Moscow, Russia
}

(October 22, 2018)

\begin{abstract}
We consider the criterion for Bose condensation for particles in a harmonic trap. For a fixed angular momentum, the lowest energy state for a cloud of bosons with attractive interactions is the ground state of the cloud with all the angular momentum in the center-of-mass motion, and the one-particle reduced density matrix generally does not have a single large eigenvalue, but a number of them, suggesting that the state is an example of a fragmented condensate (Wilkin, Gunn, and Smith, Phys. Rev. Lett. 80, 2265 (1998)). We show that a convenient way to describe correlations in the system is by defining an internal one-particle reduced density matrix, in which the center-of-mass motion is eliminated, and that this has a single eigenvalue equal to the number of particles for the problem considered here. Our considerations indicate that care is necessary in formulating a criterion for Bose-Einstein condensation.
\end{abstract}

PACS numbers: 03.75.Fi, 05.30.Jp, 67.40.Db, 67.40.Vs

In investigations of the properties of the helium liquids, rotation has proved to be a valuable probe, and this has led to the discovery of vortex lines in liquid ${ }^{4} \mathrm{He}$ and in the superfluid phases of liquid ${ }^{3} \mathrm{He}$. In the discussion of superfluidity and Bose-Einstein condensation it is usually assumed that particles are confined in a box, with a uniform potential throughout its interior, and with infinitely high walls. This situation is very different from that encountered in studies of dilute atomic vapors in traps, where the potential is generally closer to a harmonic oscillator one. We therefore consider the properties of rotating atomic clouds of bosons at zero temperature. Among possible ways of putting angular momentum into the system are the creation of vortex lines [1 3 ] and excitation of surface modes and other internal excitations [4]. For traps with an axis of symmetry, another possibility is to excite the center-of-mass motion, which for modes having rotational quantum number $m_{z}= \pm 1$ about the symmetry axis is equivalent to excitation of the simplest surface mode. The purpose of this paper is to consider the criterion for Bose condensation in a cloud of particles in a trap.

A criterion for Bose-Einstein condensation was proposed by Penrose [5], and subsequently elaborated by Penrose and Onsager [6], and by Yang [7]. Consider the one-particle reduced density matrix. This is defined in terms of the many-body wave function $\psi\left(\mathbf{r}_{1}, \ldots \mathbf{r}_{N}\right)$, where the $\mathbf{r}_{i}$ are the coordinates of the particles, by the equation

$$
\rho^{(1)}\left(\mathbf{r}, \mathbf{r}^{\prime}\right)=\int d \mathbf{r}_{2} \ldots d \mathbf{r}_{N} \psi\left(\mathbf{r}, \mathbf{r}_{2} \ldots \mathbf{r}_{N}\right) \psi^{*}\left(\mathbf{r}^{\prime}, \mathbf{r}_{2} \ldots \mathbf{r}_{N}\right)
$$

The density matrix may be expanded in terms of its eigenfunctions $\chi_{j}(\mathbf{r})$ with eigenvalues $\lambda_{j}$ :

$$
\rho^{(1)}\left(\mathbf{r}, \mathbf{r}^{\prime}\right)=\sum_{j} \lambda_{j} \chi_{j}^{*}\left(\mathbf{r}^{\prime}\right) \chi_{j}(\mathbf{r}) .
$$

The condition generally adopted for the existence of Bose condensation is that there should be one eigenvalue that is of order the number of particles, in the limit when the number of particles tends to infinity. However, as we shall indicate below, this can be misleading.

In an instructive paper Wilkin et al. [8] considered bosons in a harmonic trap, and showed that for weak attractive forces the lowest state with a given angular momentum is one in which all the angular momentum is associated with the center-of-mass motion. This corresponds to excitation of a dipolar (magnetic quantum number $m_{z}= \pm 1$ ) surface mode of oscillation [9]. An analysis of the eigenvalues of the one-particle density matrix revealed that there is not a single large eigenvalue, but rather there can be many eigenvalues of a comparable size. This led the authors to suggest that the state of the rotating system corresponds to that of a fragmented condensate of the type proposed by Nozières and Saint James [10. However, this conclusion seems surprising, because the state is just the ground state of the cloud, which is Bose-Einstein condensed, executing center-ofmass motion. The problem is to identify a quantity that can reveal the Bose condensation.

Let us consider $\mathrm{N}$ spinless bosons in a harmonic trap. This system has the important property that the centerof-mass and relative motions of the particles are separable, and therefore the wave function may be written in the form

$$
\psi\left(\mathbf{r}_{1}, \ldots \mathbf{r}_{N}\right)=\psi_{\mathrm{cm}}(\mathbf{R}) \psi_{\mathrm{rel}}\left(\mathbf{q}_{1}, \mathbf{q}_{2}, \ldots, \mathbf{q}_{N}\right),
$$

where the center-of-mass coordinate is given by $\mathbf{R}=$ $(1 / N) \Sigma_{i=1}^{N} \mathbf{r}_{i}$ and $\mathbf{q}_{i}=\mathbf{r}_{i}-\mathbf{R}$ is the coordinate of particle 
$i$ relative to the center of mass. Note that the definition of the center-of-mass coordinate implies that only $N-1$ of the $\mathbf{q}_{i}$ are independent. Let us now introduce a quantity which we call the internal density matrix

$$
\begin{aligned}
\rho_{\text {int }}\left(\mathbf{q}_{i}, \mathbf{q}_{i}^{\prime}\right) & =\int d \mathbf{R} \psi\left(\left\{\mathbf{q}_{i}+\mathbf{R}\right\}\right) \psi^{*}\left(\left\{\mathbf{q}_{i}^{\prime}+\mathbf{R}\right\}\right) \\
& =\psi_{\text {rel }}\left(\left\{\mathbf{q}_{i}\right\}\right) \psi_{\text {rel }}^{*}\left(\left\{\mathbf{q}_{i}^{\prime}\right\}\right) .
\end{aligned}
$$

This has the property that the center-of-mass motion has been eliminated, and consequently it depends only on coordinates relative to the center of mass. One may also construct reduced density matrices in terms of the relative coordinates in the standard way. For example, the internal one-particle reduced density matrix is

$$
\rho_{\text {int }}^{(1)}\left(\mathbf{q}_{1}, \mathbf{q}_{1}^{\prime}\right)=\int d \mathbf{q}_{2} \ldots d \mathbf{q}_{N-1} \rho_{\text {int }}\left(\mathbf{q}_{i}, \mathbf{q}_{i}^{\prime}\right) .
$$

Consequently the internal density matrix for the lowest energy state of a cloud of bosons with weak attractive interactions and nonzero angular momentum in a harmonic trap is the same as for the ground state in the absence of rotation. It has one large eigenvalue which is equal to $N$, to within terms of unity, the deviation from $N$ being due to the constraint that the sum of the coordinates relative to the center of mass must vanish.

Our discussion indicates that caution is required in formulating a criterion for Bose-Einstein condensation in finite systems, and that the one generally used can be misleading. For systems in harmonic traps the internal density matrix introduced above can be a useful concept, because it removes the effects of the center-of-mass motion which, for a rotating cloud, tends to spread out the weight of the usual one-particle reduced density matrix over a number of states.

We are grateful to Ben Mottelson for helpful conversations.

[1] L. P. Pitaevskii, Zh. Eksp. Teor. Fiz. 40, 646 (1961) [Sov. Phys.-JETP 13, 451 (1961)]; E. P. Gross, Nuovo Cimento 20, 454 (1961).

[2] G. Baym and C. J. Pethick, Phys. Rev. Lett. 76, 6 (1996).

[3] F. Dalfovo and S. Stringari, Phys. Rev. A 53, 2477 (1996).

[4] S. Stringari, Phys. Rev. Lett. 77, 2360 (1996).

[5] O. Penrose, Phil. Mag. 42, 1373 (1951).

[6] O. Penrose and L. Onsager, Phys. Rev. 104, 576 (1956).

[7] C. N. Yang, Rev. Mod. Phys. 34, 694 (1962).

[8] N. K. Wilkin, J. M. F. Gunn, and R. A. Smith, Phys. Rev. Lett. 80, 2265 (1998).

[9] The situation is different for repulsive interactions. The dipolar surface mode is still an eigenstate, but its energy is higher than that of other modes. For small angular momentum the ground state for a given angular momentum is obtained for weak coupling $\left(N a / a_{\text {osc }} \ll 1\right.$, where $a$ is the scattering length for two-body collisions and $a_{\text {osc }}$ is the usual characteristic length scale for the harmonic oscillator) by adding quadrupolar $\left(m_{z}= \pm 2\right)$ surface excitations to the non-rotating ground state (B. Mottelson, Phys. Rev. Lett. 83, 2695 (1999), G. M. Kavoulakis, B. Mottelson, and C. J. Pethick, (in preparation)). As the repulsive coupling increases, the optimal excitations become surface modes with higher and higher values of $\left|m_{z}\right|$. The nature of the lowest energy state for a fixed non-zero angular momentum therefore changes discontinuously as the coupling passes through zero.

[10] P. Nozières and D. Saint James, J. Phys. (Paris) 43, 1133 (1982). 